\section{Cover Page Logo of the Neuroscience Forum}

\section{To the Editor:}

Congratulations to Neuroscience Forum, Institute of Medicine for the successful launching of the journal. I got an opportunity to go through the July 2004 issue volume 1 number 2 of your journal. Along with other articles I found brain plasticity and human adaptation: a political perspective for Nepal, case studies and historical vignette extremely interesting and highly informative.

In addition to them, I was very delighted to see the complex but highly integrated logo of the journal on its cover page. It is indeed a great artwork by Dr. S. Shilpakar. But the use of two snakes entwined around a shaft for the medical symbol, called the Caduceus of Hermes, did not satisfy me as did the rest of the contents of the journal. The medical symbol should have a single snake wrapped around the shaft of Asklepios, known as the Asklepian, which is widely used around the world as the medical symbol. The caduceus of Hermes has been used in some US medical and public health institutions like the US public health service and a batch of the US medical army corps. It was not popular before 1902 when US medical army corps adopted caduceus of Hermes as the medical symbol ${ }^{1}$. But most of the medical institutions in the US, (e.g. American Medical Education, National Library of Medicine) and the rest of the world adopt the Asklepios as their medical symbol. Historically and mythlogically, Asklepios is known as the Greek god of medicine. Asklepios has also been mentioned in the writings of Homer as a mortal physician who performed heroic acts of healing on the battlefield. He has been well known as a ' blameless physician.' In contrast, Hermes is regarded as the deity of wealth and commerce, areas that is famous for dishonesty. Moreover his duty was to lead the souls of the dead to the underworld which contrasts with the deeds of the healers or helpers. So, respecting the history and mythology and still following current trends, we should be using the sign of Asklepios not the caduceus of Hermes as the medical symbol. Asklepios deserves the right because, along with other medical mythological figures, Asklepios has been mentioned in Hippocratic oath. ${ }^{2}$ I hope it would be good if we could use the Asklepian in this journal's logo too.

Lastly, I am a medical student. I, along with my eager friends, am willing to contribute to the journal; it would be great of you and a source of motivation and a pioneering deed for us, medical students if you could provide a page or a column of around 1000-1500 words in the journal.

\section{Laxmi Vilas Ghimire}

Institute of Medicine

Maharajgunj,

Kathmandu, Nepal

\section{References}

1. Robert A. Wilcox, Emma M. Whitham. The Symbol of Modern Medicine: Why One Snake Is More
Than Two. Ann Intern Med 138:673-677, 2003

2. von Staden H. "In a pure and holy way": personal and professional conduct in the Hippo cratic Oath? J Hist Med Allied Sci 51:404-37, 1996

\section{Response 1:}

Thank you very much for your comment and the homework well done. It is very interesting to know the elaborate history behind the two serpents and one serpent. In answer to your question concerning my choice of the two serpents instead of one when designing the TUTH Neuroscience Logo, I had simply taken part of the Nepal Medical Association Logo and incorporated in it. This was done to make it clear that we are a part of the Nepal Medical Association. Moreover, I had thought that the inclusion of both sexes, the male and female serpents, was more natural. It had also come to my mind that the intertwining serpents resembled the double helical model of a DNA molecule. In brief, the selection was merely based on the aforementioned facts, not on any biases and has no association with the historical background.

\section{Sushil K. Shilpakar, MS \\ Division of Neurosurgery \\ TU Teaching Hospital \\ Kathmandu, Nepal}

\section{Response 2:}

I appreciate your interest in our journal and I extend my sincere thanks for your encouraging words. The whole goal of publishing this journal right from the inception has been to publish good articles covering all areas of neuroscience and one or two thought-provoking articles on general fields. I believe that, with the enthusiastic support of people like you, we will be able to achieve this goal.

As you may have noticed, the journal is becoming more refined with each issue. New ideas and suggestions are always welcome with the aim of further improving the journal. I am pleased to inform you that your suggestion of including a Medical Student's Page has been taken very positively by our Editorial Board and most likely we will be starting this from the July 2005 issue.

\section{Mohan R. Sharma, MS \\ Editor \\ Nepal Journal of Neuroscience}

\section{Tumor Angiogenesis}

\section{To the Editor:}

I read with interest the article by Singh "Tumor angiogenesis: Clinical Implications" (Nepal Journal of Neuroscience 1:60-61, 2004). ${ }^{4}$ Angiogenesis has been the main mechanism in tumor growth and many other nonmalignant diseases e.g. diabetic retinopathy, retrolental fibroplasias and arthritis. ${ }^{4}$ So the clinical implications of angiogenesis are of course unlimited and are yet to be 
explored. The High Altitude Cerebral Edema (HACE), a severe form of Acute Mountain Sickness (AMS), is one of these. The physiological control of angiogenesis involves a complex interplay between endogenous positive and negative regulators of blood vessel growth. The exact process of AMS and HACE is still unclear. The hypoxiainduced cerebral vasodilatation or its effectors, such as nitric oxide, most probably produce the headache, perhaps through the activation of the trigeminovascular system. The neuroimaging technique demonstrates vasogenic edema. The latest update is that on ascent to high altitudes, all people have swelling of the brain. ${ }^{3}$ This is the reason why the asymptomatic brain tumors of the lowlanders get symptoms (focal neurological signs and cranial nerve palsies) on ascent to high altitudes. ${ }^{1}$ The moderate-to-severe AMS or HACE, the Hemodynamic factors such as sustained vasodilatation, impaired cerebral autoregulation, and elevated cerebral capillary pressure may contribute to the formation of edema but they are not the only processes to lead to the HACE. As there is hypobaric hypoxia at high altitudes, the hypoxia-induced biochemical alteration of the blood-brain barrier (BBB) is also important. Possible mediators, triggered by endothelial activation, include Vascular Endothelial Growth Factor(VEGF), inducible nitric oxide synthase (iNOS), and bradykinin. ${ }^{3}$ The gene expression and production of VEGF, the potent promoter of capillary leakage, is upregulated by hypoxia and may play a part in the development of AMS and HACE. There is evidence that the VEGF causes hypoxia-induced increase of vascular leakage in the brains of mice. ${ }^{2}$ So the angiogenesis produces new capillary growth brought about by VEGF which increases the BBB permeability leading to the brain swelling, the basis of HACE. More importantly the dexamethasone, a steroid, which is very effective in the treatment of HACE, blocks the angiogenesis. The inhibition of angiogenesis is the mechanism of action of dexamethasone in efficiently shrinking the brain tumors. ${ }^{1}$ Various studies have shown that angiogenesis inhibition can suppress tumor growth and metastasis. There a number of molecules that have been designed to target VEGF to treat tumor angiogenesis. If success comes that will not only treat tumor angiogenesis but also treat nonmalignant angiogenesis including HACE.

\section{Matiram Pun}

Institute of Medicine

Maharajgunj,

Kathmandu, Nepal

\section{References}

1. Basnyat, B. High Altitude Cerebral and Pulmonary Edema. Journal of Institute of Medicine 26:22-32, 2004
2. Basnyat B, Murdoch D: High-altitude Illness. The Lancet 361:1967-1974, 2003

3. Hackett PH, Roach RC: High-Altitude Illness. N Engl J Med 345: 107-114, 2001

4. Singh, Y: Tumour Angiogenesis: Clinical Implications. Nepal Journal of Neuroscience1: 61-63, 2004

\section{Response:}

Thank you very much for your enthusiastic concern on angiogenesis in neoplastic as well as non-neoplastic conditions, particularly high altitude cerebral edema (HACE). Physiologically, angiogenesis is a very strictly regulated process which results in a balance between stimulatory (angiogenic) and inhibitory (angiostatic) factors to control the correct development of blood vessels. As you have pointed out the evidence linking HACE to angiogenesis is that dexamethasone, an effective inhibitor of angiogenesis, has demonstrated unique success in preventing and treating HACE. Because, in HACE, petechial hemorrhages are seen in the nerve layers of the retina and similar changes have been described throughout the brain. ${ }^{3}$ Formation of cerebral edema caused by vascular leakage is a major problem in various injuries of the CNS, including high-altitude illness. It has been suggested that hypoxia is an important pathogenic factor for the induction of vascular leakage in the brain. ${ }^{2}$ Hypoxia inducible factor 1 is the best characterized transcription regulator of the vascular endothelial growth factor (VEGF) gene. ${ }^{1}$ VEGF is responsible for hypoxia induced vascular leakage following tissue hypoxia. These findings may provide a novel treatment of cerebral edema.

\author{
Yogendra P, Singh, $\mathrm{MD}, \mathrm{PhD}$ \\ Department of Surgery \\ TU Teaching Hospital \\ Kathmandu, Nepal
}

\section{References}

1. Carineliet P, Collen D: Role of vascular endothelial growth factor and vascular endothelial growth factor receptors in vascular development. Curr Top Microbiol Immunol 237: 133-158, 1999

2. Schoch HJ, Fischer S, Marti HH: Hypoxia induced vascular endothelial factor expression causes vascular leakage in the brain. Brain 125:2549-2557, 2002

3. $\mathrm{XuF}$, Severinghaus JW: Rat brain VEGF expression in alveolar hypoxia: possible role in high altitude cerebral edema. J Appl Physiol 85:53-57, 1998 